\title{
Multi-band Gradient Component Pattern (MGCP): A New Statistical Feature for Face Recognition
}

\author{
Yimo Guo ${ }^{1,2}$, Jie Chen ${ }^{1}$, Guoying Zhao ${ }^{1}$, Matti Pietikäinen ${ }^{1}$, and Zhengguang $\mathrm{Xu}^{2}$ \\ ${ }^{1}$ Machine Vision Group, Department of Electrical and Information Engineering, \\ University of Oulu, P.O. Box 4500, FIN-90014, Finland \\ ${ }^{2}$ School of Information Engineering, University of Science and Technology Beijing, \\ Beijing, 100083, China
}

\begin{abstract}
A feature extraction method using multi-frequency bands is proposed for face recognition, named as the Multi-band Gradient Component Pattern (MGCP). The MGCP captures discriminative information from Gabor filter responses in virtue of an orthogonal gradient component analysis method, which is especially designed to encode energy variations of Gabor magnitude. Different from some well-known Gabor-based feature extraction methods, MGCP extracts geometry features from Gabor magnitudes in the orthogonal gradient space in a novel way. It is shown that such features encapsulate more discriminative information. The proposed method is evaluated by performing face recognition experiments on the FERET and FRGC ver 2.0 databases and compared with several state-of-the-art approaches. Experimental results demonstrate that MGCP achieves the highest recognition rate among all the compared methods, including some well-known Gabor-based methods.
\end{abstract}

\section{Introduction}

Face recognition receives much attention from both research and commercial communities, but it remains challenging in real applications. The main task of face recognition is to represent object appropriately for identification. A well designed representation method should extract discriminative information effectively and improve recognition performance. This depends on deep understanding of the object and recognition task itself. Especially, there are two problems involved: (i) what representation is desirable for pattern recognition; (ii) how to represent the information contained in both neighborhood and global structure. In the last decades, numerous face recognition methods and their improvements have been proposed. These methods can be generally divided into two categories: holistic matching methods and local matching methods. Some representative methods are Eigenfaces [1], Fisherfaces [2], Independent Component Analysis [3], Bayesian [4], Local Binary Pattern (LBP) [5,6], Gabor features $[7,12,13]$, gradient magnitude and orientation maps [8], Elastic Bunch Graph Matching [9] and so on. All these methods exploit the idea to obtain features using an operator and build up a global representation or local neighborhood representation.

Recently, some Gabor-based methods that belong to local matching methods have been proposed, such as the local Gabor binary pattern (LGBPHS) [10], enhanced local 
Gabor binary pattern (ELGBP) [11] and the histogram of Gabor phase patterns (HGPP) [12]. LGBPHS and ELGBP explore information from Gabor magnitude, which is a commonly used part of the Gabor filter response, by applying local binary pattern to Gabor filter responses. Similarly, HGPP introduced LBP for further feature extraction from Gabor phase that was demonstrated to provide useful information. Although LBP is an efficient descriptor for image representation, it is good at capturing neighborhood relationships from original images in the spatial domain. To process multi-frequency bands responses using LBP would increase complexity and lose information.

Therefore, to improve the recognition performance and efficiency, we propose a new method to extract discriminative information especially from Gabor magnitude. Useful information would be extracted from Gabor filter responses in an elaborate way by making use of the characteristics of Gabor magnitude. In detail, based on Gabor function and gradient theory, we design a Gabor energy variation analysis method to extract discriminative information. This method encodes Gabor energy variations to represent images for face recognition. The gradient orientations are selected in a hierarchical fashion, which aims to improve the capability of capturing discriminative information from Gabor filter responses. The spatially enhanced representation is finally described as the combination of these histogram sequences at different scales and orientations. From experiments conducted on the FERET database and FRGC ver 2.0 database, our method is shown to be more powerful than many other methods, including some well-known Gabor-based methods.

The rest of this paper is organized as follows. In Section 2, the image representation method for face recognition is presented. Experiments and result analysis are reported in Section 3. Conclusions are drawn in Section 4.

\section{Multi-band Gradient Component Pattern (MGCP)}

Gabor filters have been widely used in pattern recognition because of their multiscale, multi-orientation, multi-frequency and processing capability. Most of the proposed Gabor-based methods take advantage of Gabor magnitude to represent face images. Although Gabor phase was demonstrated to be a good compensation to the magnitude, information should be exploited elaborately from the phase in order to avoid the sensitivity to local variations [11]. Considering that the Gabor magnitude part varies slowly with spatial position and contains enough discriminative information for classification, we extract features from this part of Gabor filter responses. In detail, features are obtained from Gabor responses using an energy variation analysis method. The gradient component is adopted here because: (i) gradient magnitudes contain intensity variation information; (ii) gradient orientations of neighborhood pixels contain rich directional information and are insensitive to illumination and pose variations [15]. In this way, features are described as histogram sequences explored from Gabor filter responses at each scale and orientation.

\subsection{Multi-frequency Bands Feature Extraction Method Using Gabor Filters}

Gabor function is biologically inspired, since Gabor like receptive fields have been found in the visual cortex of primates [16]. It acts as low-level oriented edge and texture discriminator and is sensitive to different frequencies and scale information. 
These characteristics raise considerable interests for researchers to extensively exploit its properties. Gabor wavelets are biologically motivated convolution kernels in the shape of plane waves restricted by a Gaussian envelope function [17]. The general form of a 2D Gabor wavelet is defined as:

$$
\Psi_{u, v}(z)=\left(\left\|k_{u, v}\right\|^{2} / \sigma^{2}\right) \exp \left(-\left\|k_{u, v}\right\|^{2}\|z\|^{2} / 2 \sigma^{2}\right)\left[\exp \left(i k_{u, v} z\right)-\exp \left(-\sigma^{2} / 2\right)\right],
$$

where $u$ and $v$ define the orientation and scale of Gabor kernels. $\sigma$ is a parameter to control the scale of Gaussian. $\vec{k}_{u, v}$ is a $2 \mathrm{D}$ wave vector whose magnitude and angle determine the scale and orientation of Gabor kernel respectively. In most cases, Gabor wavelets at five different scales $v:\{0, \ldots 4\}$ and eight orientations $u:\{0, \ldots 7\}$ are used $[18,19,20]$. The Gabor wavelet transformation of an image is the convolution of the image with a family of Gabor kernels, as defined by:

$$
G_{u, v}(z)=I(z) * \Psi(z),
$$

where $z=(x, y)$. The operator $*$ is the convolution operator. $G_{u, v}(z)$ is the convolution corresponding to Gabor kernels at different scales and orientations. The Gabor magnitude is defined as:

$$
M_{u, v}(z)=\sqrt{\operatorname{Re}\left(G_{u, v}(z)\right)^{2}+\operatorname{Im}\left(G_{u, v}(z)\right)^{2}},
$$

where $\operatorname{Re}(\cdot)$ and $\operatorname{Im}(\cdot)$ denote the real and imaginary part of Gabor transformed image respectively, as shown in Fig. 1. In this way, 40 Gabor magnitudes are calculated to form the representation. The visualization of Gabor magnitudes are shown in Fig. 2.

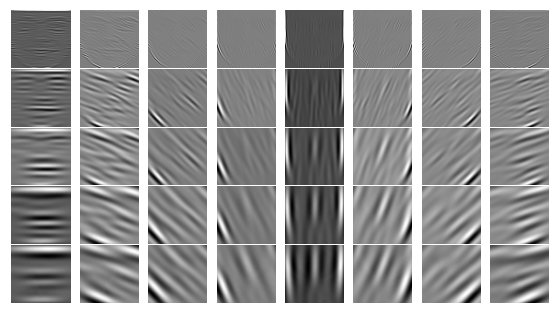

(a)
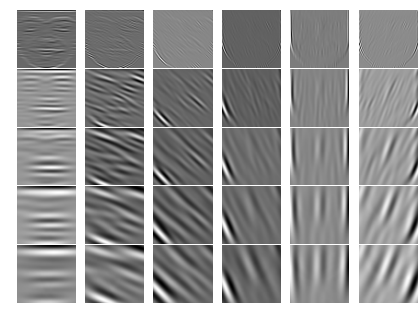

(b)

Fig. 1. The visualization of a) the real part and b) imaginary part of a Gabor transformed image
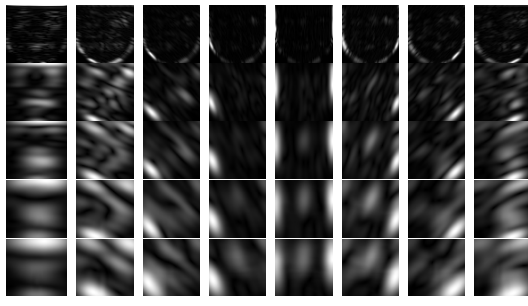

Fig. 2. The visualization of Gabor magnitudes 


\subsection{Orthogonal Gradient Component Analysis}

There has been some recent work makes use of gradient information in object representation [21,22]. As Gabor magnitude part varies slowly with spatial position and embodies energy information, we explore Gabor gradient components for representation. Motivated by using the Three Orthogonal Planes to encode texture information [23], we select orthogonal orientations (horizontal and vertical) here. This is mainly because Gabor gradient is defined based on Gaussian function, which is not declining at exponential speed as in Gabor wavelets. These two orientations are selected as: (i) the gradient of orthogonal orientations could encode more variations with less correlation; (ii) less time is needed to calculate two orientations than in some other Gaborbased methods, such as LGBPHS and ELGBP, which calculate eight neighbors to capture discriminative information from Gabor magnitude.

Given an image $I(z)$, where $z=(x, y)$ indicates the pixel location. $G_{u, v}(z)$ is the convolution corresponding to the Gabor kernel at scale $v$ and orientation $u$. The gradient of $G_{u, v}(z)$ is defined as:

$$
\left|\nabla_{d} G_{u, v}(z)\right|=\left(\partial G_{u, v} / \partial x\right) \hat{i}+\left(\partial G_{u, v} / \partial y\right) \hat{j} .
$$

Equation 4 is the set of vectors pointing at appointed directions of increasing values of $G_{u, v}(z)$. The $\partial G_{u, v} / \partial x$ corresponds to differences in the horizontal (row) direction, while the $\partial G_{u, v} / \partial y$ corresponds to differences in the vertical (column) direction. The $x$-and $y$-gradient components of Gabor filter responses are calculated at each scale and orientation. The gradient components are shown in Fig. 3.

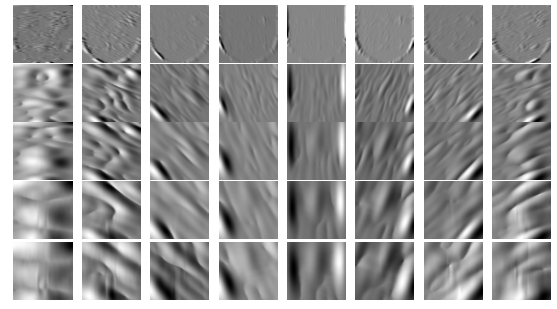

(a)
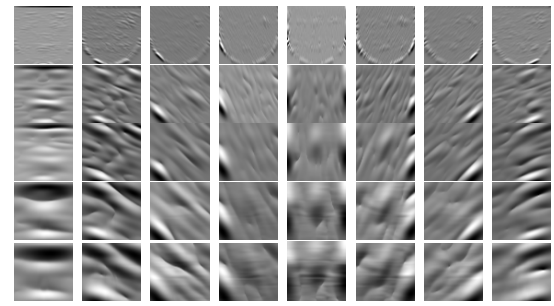

(b)

Fig. 3. The gradient components of Gabor filter responses at different scales and orientations. a) $\mathrm{x}$-gradient components in horizontal direction; b) $\mathrm{y}$-gradient components in vertical direction.

The histograms (256 bins) of $x$-and $y$-gradient components of Gabor responses at different scales and orientations are calculated and concatenated to form the representation. From Equations 3 and 4, we can see that MGCP actually encodes the information of Gabor energy variations in orthogonal orientations, which contains very discriminative information as shown in Section 4.

Considering Gabor magnitude provides useful information for face recognition, we propose MGCP to encode Gabor energy variations for face representation. However, a single histogram suffers from losing spatial structure information. Therefore, images 
are decomposed into non-overlapping sub-regions, from which local features are extracted. To capture both the global and local information, all these histograms are concatenated to an extended histogram for each scale and orientation. Examples of concatenated histograms are illustrated in Fig. 4 (c) when images are divided into non-overlapping $4 \times 4$ sub-regions. The $4 \times 4$ decomposition will result in a little weak feature but can further demonstrate the performance of our method. Fig. 4 (b) illustrates the MGCP $(u=90, v=5.47)$ of four face images for two subjects. The $u$ and $v$ are selected randomly. The capability of these discriminative patterns could be observed from histogram distances, listed in Table 1.

$\mathrm{S} 11$
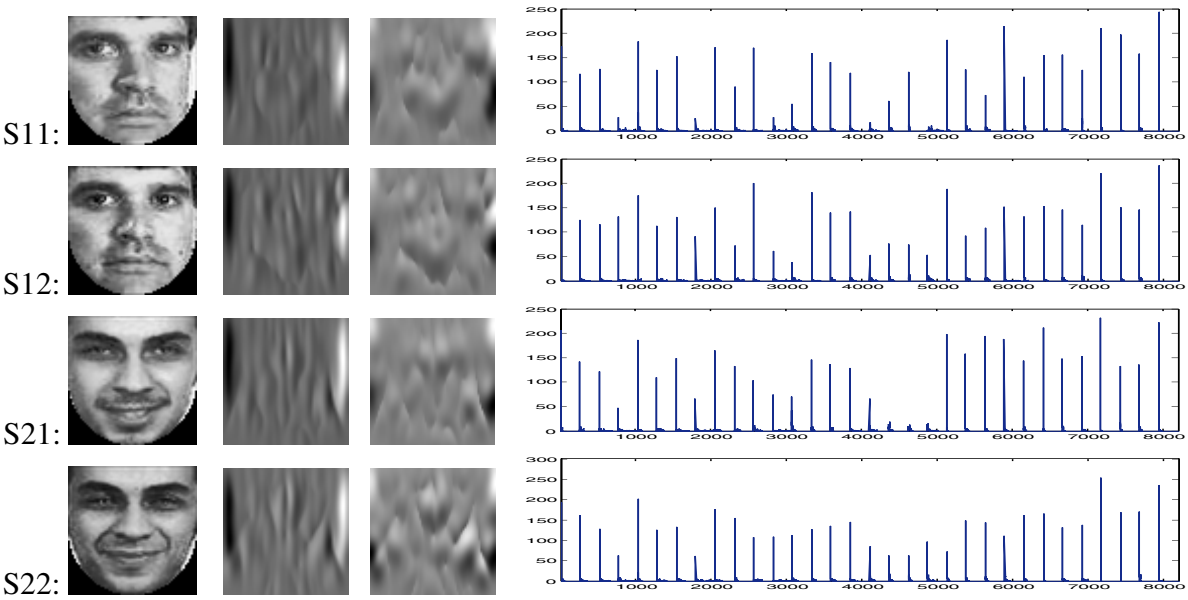

(a)

(b)

(c)

Fig. 4. MGCP ( $u=90, v=5.47)$ of four images for two subjects. a) The original face images; b) the visualization of gradient components of Gabor filter responses; c) the histograms of all subregions when images are divided into non-overlapping $4 \times 4$ sub-regions. The input images from the FERET database are cropped and normalized to the resolution of $64 \times 64$ using eye coordinates provided.

Table 1. The histogram distances of four images for two subjects using MGCP

\begin{tabular}{ccccc}
\hline Subjects & S11 & S12 & S21 & S22 \\
\hline S11 & 0 & 4640 & 5226 & 5536 \\
S12 & -- & 0 & 4970 & 5266 \\
S21 & -- & -- & 0 & 4708 \\
S22 & -- & -- & -- & 0 \\
\hline
\end{tabular}

\section{Experiments}

The proposed method is tested on the FERET database and FRGC ver 2.0 database $[24,25]$. The classifier is the simplest classification scheme: nearest neighbour classifier in image space with Chi square statistics as the similarity measure. 


\subsection{Experiments on the FERET Database}

To conduct experiments on the FERET database, we use the same Gallery and Probe sets as the standard FERET evaluation protocol. For the FERET database, we use Fa as gallery, which contains 1196 frontal images of 1196 subjects. The probe sets consist of Fb, Fc, Dup I and Dup II. Fb contains 1195 images of expression variations, Fc contains 194 images taken under different illumination conditions, Dup I has 722 images taken later in time and Dup II (a subset of Dup I) has 234 images taken at least one year after the corresponding Gallery images. Using $\mathrm{Fa}$ as the gallery, we design the following experiments: (i) use $\mathrm{Fb}$ as probe set to test the efficiency of the method against facial expression; (ii) use Fc as probe set to test the efficiency of the method against illumination variation; (iii) use Dup I as probe set to test the efficiency of the method against short time; (iv) use Dup II as probe set to test the efficiency of the method against longer time. All images in the database are cropped and normalized to the resolution of $64 \times 64$ using eye coordinates provided. Then they are divided into $4 \times 4$ non-overlapping sub-regions. To validate the superiority of our method, recognition rates of MGCP and some state-of-the-art methods are listed in Table 2.

Table 2. The recognition rates of different methods on the FERET database probe sets (\%)

\begin{tabular}{lcccc}
\hline Methods & \multicolumn{4}{c}{ FERET Probe Sets } \\
& Fb & Fc & Dup I & Dup II \\
\hline PCA [1] & 85.0 & 65.0 & 44.0 & 22.0 \\
UMDLDA [26] & 96.2 & 58.8 & 47.2 & 20.9 \\
Bayesian, MAP [4] & 82.0 & 37.0 & 52.0 & 32.0 \\
LBP [5] & 93.0 & 51.0 & 61.0 & 50.0 \\
LBP_W [5] & 97.0 & 79.0 & 66.0 & 64.0 \\
LGBP_Pha [11] & 93.0 & 92.0 & 65.0 & 59.0 \\
LGBP_Pha_W[11] & 96.0 & 94.0 & 72.0 & 69.0 \\
LGBP_Mag [10] & 94.0 & 97.0 & 68.0 & 53.0 \\
LGBP_Mag_W [10] & 98.0 & 97.0 & 74.0 & 71.0 \\
ELGBP (Mag + Pha) [11] & 97.0 & 96.0 & 77.0 & 74.0 \\
MGCP & 97.4 & 97.3 & 77.8 & 73.5 \\
\hline
\end{tabular}

As seen from Table 2, the proposed method outperforms LBP, LGBP_Pha and their corresponding methods with weights. The MGCP also outperforms LGBP_Mag that represents images using Gabor magnitude information. Moreover, from experimental results of Fa-X (X: Fc, Dup I and Dup II), MGCP without weights performs better than LGBP_Mag with weights. From experimental results of $\mathrm{Fa}-\mathrm{Y}$ (Y: Fb, Fc and Dup I), MGCP performs even better than ELGBP that combines both the magnitude and phase patterns of Gabor filter responses.

\subsection{Experiments on the FRGC Ver 2.0 Database}

To further evaluate the performance of the proposed method, we conduct experiments on the FRGC version 2.0 database which is one of the most challenging databases [25]. The face images are normalized and cropped to the size of $120 \times 120$ using eye coordinates provided. Some samples are shown in Fig. 5. 


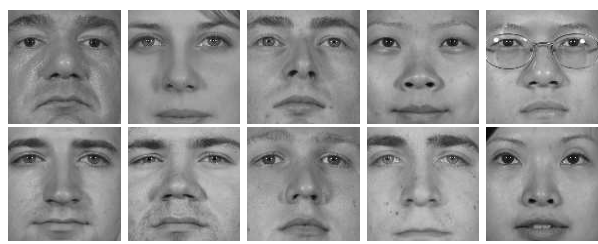

Fig. 5. Face images from FRGC 2.0 database

In FRGC 2.0 database, there are 12776 images taken from 222 subjects in the training set and 16028 images in the target set. We conduct Experiment 1 and Experiment 4 protocols to evaluate the performance of different approaches. In Experiment 1, there are 16028 query images taken under the controlled illumination condition. The goal of Experiment 1 is to test the basic recognition ability of approaches. In Experiment 4, there are 8014 query images taken under the uncontrolled illumination condition. Experiment 4 is the most challenging protocol in FRGC because the uncontrolled large illumination variations bring significant difficulties to achieve high recognition rate. The experimental results on the FRGC 2.0 database in Experiment 1 and 4 are evaluated by Receiving Operator Characteristics (ROC), which is face verification rate (FVR) versus false accept rate (FAR). Tables 3 and 4 list the performance of different approaches on face verification rate $(\mathrm{FVR})$ at false accept rate (FAR) of $0.1 \%$ in Experiment 1 and 4.

From experimental results listed in Table 3, MGCP achieves the best performance, which demonstrates its basic abilities in face recognition. Table 4 exhibits results of MGCP and two well-known approaches: BEE Baseline and LBP. MGCP is also compared with some recently proposed methods and the results are listed in Table 5. The database used in experiments for Gabor + FLDA, LGBP, E-GV-LBP, GV-LBP-TOP are reported to be a subset of FRGC 2.0, while the whole database is used in experiments for UCS and MGCP. It is observed from Table 4 and 5 that MGCP could overcome uncontrolled condition variations effectively and improve face recognition performance.

Table 3. The FVR value of different approaches at FAR $=0.1 \%$ in Experiment 1 of the FRGC 2.0 database

\begin{tabular}{lccc}
\hline Methods & \multicolumn{3}{c}{ FVR at FAR $=0.1 \%$ (in \%) } \\
& ROC 1 & ROC 2 & ROC 3 \\
\hline BEE Baseline [25] & 77.63 & 75.13 & 70.88 \\
LBP [5] & 86.24 & 83.84 & 79.72 \\
MGCP & 97.52 & 94.08 & 92.57 \\
\hline
\end{tabular}

Table 4. The FVR value of different approaches at FAR $=0.1 \%$ in Experiment 4 of the FRGC 2.0 database

\begin{tabular}{lccc}
\hline Methods & \multicolumn{3}{c}{ FVR at FAR $=0.1 \%$ (in \%) } \\
& ROC 1 & ROC 2 & ROC 3 \\
\hline BEE Baseline [25] & 17.13 & 15.22 & 13.98 \\
LBP [5] & 58.49 & 54.18 & 52.17 \\
MGCP & 76.08 & 75.79 & 74.41 \\
\hline
\end{tabular}


Table 5. ROC 3 on the FRGC 2.0 in Experiment 4

\begin{tabular}{lc}
\hline Methods & ROC 3, FVR at FAR $=0.1 \%$ (in \%) \\
BEE Baseline [25] & 13.98 \\
Gabor + FLDA [27] & 48.84 \\
LBP [27] & 52.17 \\
LGBP [27] & 52.88 \\
E-GV-LBP [27] & 53.66 \\
GV-LBP-TOP [27] & 54.53 \\
UCS [28] & 69.92 \\
MGCP & 74.41 \\
\hline
\end{tabular}

\section{Conclusions}

To extend traditional use of multi-band responses, the proposed feature extraction method encodes Gabor magnitude gradient component in an elaborate way, which is different from some previous Gabor-based methods that directly apply some proposed feature extraction methods on Gabor filter responses. Especially, the gradient orientations are organized in a hierarchical fashion. Experimental results show that orthogonal orientations could improve the capability to capture energy variations of Gabor responses. The spatial histograms of multi-frequency bands gradient component pattern at each scale and orientation are finally concatenated to represent face images, which could encode both the structure and local information. From experimental results conducted on the FERET and FRGC 2.0, it is observed that the proposed method is insensitive to many variations, such as illumination and pose. The experimental results also demonstrate its efficiency and validity in face recognition.

Acknowledgments. The authors would like to thank the Academy of Finland for their support to this work.

\section{References}

1. Turk, M., Pentland, A.: Eigenfaces for recognition. Journal of Cognitive Neuroscience 3(1), 71-86 (1991)

2. Belhumeur, P.N., Hespanha, J.P., Kriegman, D.J.: Eigenfaces vs. Fisherfaces: Recognition using class specific linear projection. IEEE Transactions on Pattern Analysis and Machine Intelligence 19(7), 711-720 (1997)

3. Bartlett, M.S., Movellan, J.R., Sejnowski, T.J.: Face recognition by independent component analysis. IEEE Transactions on Neural Networks 13(6), 1450-1464 (2002)

4. Phillips, P., Syed, H., Rizvi, A., Rauss, P.: The FERET evaluation methodology for facerecognition algorithms. IEEE Transactions on Pattern Analysis and Machine Intelligence 22(10), 1090-1104 (2000)

5. Ahonen, T., Hadid, A., Pietikäinen, M.: Face recognition with local binary patterns. In: Pajdla, T., Matas, J(G.) (eds.) ECCV 2004. LNCS, vol. 3021, pp. 469-481. Springer, Heidelberg (2004)

6. Ahonen, T., Hadid, A., Pietikäinen, M.: Face description with local binary pattern. IEEE Transactions on Pattern Analysis and Machine Intelligence 28, 2037-2041 (2006) 
7. Daugman, J.G.: Two-dimensional spectral analysis of cortical receptive field problems. Vision Research (20), 847-856 (1980)

8. Lowe, D.: Object recognition from local scale-invariant features. In: Conference on Computer Vision and Pattern Recognition, pp. 1150-1157 (1999)

9. Wiskott, L., Fellous, J.-M., Kruger, N., Malsburg, C.v.d.: Face recognition by Elastic Bunch Graph Matching. IEEE Transactions on Pattern Analysis and Machine Intelligence 19(7), 775-779 (1997)

10. Zhang, W., Shan, S., Gao, W., Chen, X., Zhang, H.: Local Gabor Binary Pattern Histogram Sequence (LGBPHS): a novel non-Statistical model for face representation and recognition. In: International Conference on Computer Vision, pp. 786-791 (2005)

11. Zhang, W., Shan, S., Chen, X., Gao, W.: Are Gabor phases really useless for face recognition? In: International Conference on Pattern Recognition, vol. 4, pp. 606-609 (2006)

12. Zhang, B., Shan, S., Chen, X., Gao, W.: Histogram of Gabor Phase Pattern (HGPP): A novel object representation approach for face recognition. IEEE Transactions on Image Processing 16(1), 57-68 (2007)

13. Lyons, M.J., Budynek, J., Plante, A., Akamatsu, S.: Classifying facial attributes using a 2D Gabor wavelet representation and discriminant analysis. In: Conference on Automatic Face and Gesture Recognition, pp. 1357-1362 (2000)

14. Liu, C., Wechsler, H.: Gabor feature based classification using the enhanced fisher linear discriminant model for face recognition. IEEE Transactions on Image Processing 11, $467-$ 476 (1997)

15. Chen, H., Belhumeur, P., Jacobs, D.W.: In search of illumination invariants. In: Conference on Computer Vision and Pattern Recognition, pp. 254-261 (2000)

16. Daniel, P., Whitterridge, D.: The representation of the visual field on the cerebral cortex in monkeys. Journal of Physiology 159, 203-221 (1961)

17. Wiskott, L., Fellous, J.-M., Kruger, N., Malsburg, C.v.d.: Face recognition by Elastic Bunch Graph Matching. In: Intelligent Biometric Techniques in Fingerprint and Face Recognition, ch. 11, pp. 355-396 (1999)

18. Field, D.: Relations between the statistics of natural images and the response properties of cortical cells. Journal of the Optical Society of America A: Optics Image Science and Vision 4(12), 2379-2394 (1987)

19. Jones, J., Palmer, L.: An evaluation of the two-dimensional Gabor filter model of simple receptive fields in cat striate cortex. Journal of Neurophysiology 58(6), 1233-1258 (1987)

20. Burr, D., Morrone, M., Spinelli, D.: Evidence for edge and bar detectors in human vision. Vision Research 29(4), 419-431 (1989)

21. Lowe, D.: Distinctive Image Features from Scale-Invariant Keypoints. International Journal of Computer Vision 60(2), 91-110 (2004)

22. Dalal, N., Triggs, B.: Histograms of oriented gradients for human detection. In: Conference on Computer Vision and Pattern Recognition, vol. 1, pp. 886-893 (2005)

23. Zhao, G., Pietikäinen, M.: Dynamic texture recognition using local binary patterns with an application to facial expressions. IEEE Transactions on Pattern Analysis and Machine Intelligence 29(6), 915-928 (2007)

24. Phillips, P., Wechsler, H., Huang, J., Rauss, P.: The FERET database and evaluation procedure for face recognition algorithms. Image and Vision Computing 16(5), 295-306 (1998)

25. Phillips, P.J., Flynn, P.J., Scruggs, T., Bowyer, K.W., Chang, J., Hoffman, K., Marques, J., Min, J., Worek, W.: Overview of the face recognition grand challenge. In: IEEE Conference on Computer Vision and Pattern Recognition, pp. 947-954 (2005) 
26. Ravela, S., Manmatha, R.: Retrieving images by appearance. In: International Conference on Computer Vision, pp. 608-613 (1998)

27. Lei, Z., Liao, S., He, R., Pietikäinen, M., Li, S.: Gabor volume based local binary pattern for face representation and recognition. In: IEEE conference on Automatic Face and Gesture Recognition (2008)

28. Liu, C.: Learning the uncorrelated, independent, and discriminating color spaces for face recognition. IEEE Transactions on Information Forensics and Security 3(2), 213-222 (2008) 